\title{
Effect of Gum Chewing on the Recovery From Laparoscopic Colorectal Cancer Surgery
}

\author{
Duk Yeon Hwang, Ho Young Kim, Ji Hoon Kim, In Gyu Lee, Jun Ki Kim, Seung Taek Oh, Yoon Suk Lee \\ Division of Colorectal Surgery, Department of Surgery, The Catholic University of Korea School of Medicine, Seoul, Korea
}

Purpose: We aimed to examine the effect of gum chewing after laparoscopic colorectal cancer surgery.

Methods: We reviewed the medical records of patients who underwent laparoscopic colorectal cancer surgery in Incheon St. Mary's Hospital, The Catholic University of Korea School of Medicine. We divided the patients into 2 groups: group A consisted of 67 patients who did not chew gum; group B consisted of 65 patients who chewed gum. We analyzed the short-term clinical outcomes between the two groups to evaluate the effect of gum chewing.

Results: The first passage of gas was slightly earlier in group B, but the difference was not significant. However, the length of hospital stay was 6.7 days in group B, which was significantly shorter than that in group A ( 7.3 days, $\mathrm{P}=0.018$ ).

Conclusion: This study showed that length of postoperative hospital stay was shorter in the gum-chewing group. In future studies, we expect to elucidate the effect of gum chewing on the postoperative recovery more clearly.

Keywords: Chewing gum; Colorectal neoplasms; Laparoscopy; Sham feeding

\section{INTRODUCTION}

Colorectal cancer is one of the most common malignant tumors in the world. In Korea, the incidence of colorectal cancer has been increasing since 2000, as the lifestyle has become westernized. Accordingly, the number of colorectal cancer surgeries has also increased precipitously [1].

Postoperative ileus is one of the most common complications after abdominal surgery [2]. Postoperative ileus increases abdominal discomfort, pain, the number of nosocomial infections and the length of hospital stay and, as a result, can lead to an overall increase in the medical costs. The incidence of postoperative ileus in laparoscopic surgery is lower than that in conventional open surgery. However, the surgical extent and dissection area are wide

Received: June 07,2013 • Accepted: August 28, 2013

Correspondence to: : Yoon Suk Lee, M.D.

Division of Colorectal Surgery, Department of Surgery, Incheon St. Mary's Hospital, The Catholic University of Korea School of Medicine, 56 Dongsu-ro, Bupyeong-gu, Incheon 403-720, Korea

Tel: +82-32-280-6363, Fax: +82-32-280-5556

E-mail:yslee@catholic.ac.kr

(c) 2013 The Korean Society of Coloproctology

This is an open-access article distributed under the terms of the Creative Commons Attribution NonCommercial License (http://creativecommons.org/licenses/by-nc/3.0) which permits unrestricted noncommercial use, distribution, and reproduction in any medium, provided the original work is properly cited. in laparoscopic colorectal cancer surgery, which could be a risk for postoperative ileus [3]. Thus far, various treatments have been attempted to prevent and shorten the duration of ileus. Choi and O'Connell [4] reported that it was safe to start early feeding as soon as possible after conventional open colorectal surgery and that the early-feeding group had a shorter hospital stay than the late-feeding group. Stewart et al. [5] also reported that early feeding after surgery could decrease postoperative ileus and shorten hospital stay. However, approximately $20 \%$ of the patients showed difficulties with or did not tolerate early feeding [5]. Thus, as an alternative to early feeding, "sham feeding" by gum chewing was introduced and was found to lead to an early recovery of intestinal motility and shortened hospital stay [6].

Gum chewing promotes the cephalic-vagal reflex, which is activated when eating food, and it is thought to promote the secretion of hormones related to intestinal motility [7]. However, few studies have reported on the relationship between gum chewing and recovery from postoperative ileus after laparoscopic surgery. In this study, we aimed to examine the effect of gum chewing after laparoscopic colorectal cancer surgery.

\section{METHODS}

We reviewed the medical records of patients who underwent lap- 
aroscopic colorectal cancer surgery from January 2012 to November 2012 at Incheon St. Mary's Hospital, The Catholic University of Korea School of Medicine.

In this study, patients who needed to have a stoma (temporary or permanent) made or had postoperative complications such as leakage were excluded. We divided the patients into 2 groups: group A consisted of 67 patients who did not chew gum; group B consisted of 65 patients who chewed gum. Patients in the gumchewing group were asked to chew gum, starting at the first postoperative day, 3 times a day, approximately 10-20 minutes at a time, until normal feeding was resumed. Postoperative care of both groups was the same and was in accordance with the institution's clinical pathway program with the exception of gum chewing. We analyzed the short-term clinical outcomes between the two groups to evaluate the effect of gum chewing. In this study, $\mathrm{P}$ $<0.05$ was considered statistically significant.

Patients were considered appropriate for discharge if they passed flatus, passed urine freely, ambulated independently, and tolerated a diet and if pain was managed with oral medication, the patient was willing to leave the hospital and had adequate home support, and there was no evidence of postoperative complication. These factors all contributed to the length of the postoperative hospital stay, and as mentioned above, patients who had postoperative complications were excluded in this study.

\section{RESULTS}

No significant differences were observed between the 2 groups in terms of patient's demographics, American Society of Anesthesiologists physical status score, body mass index (BMI) or prior history of abdominal surgery (Table 1). The types of surgical procedures for colorectal cancer were not significantly different between the 2 groups. The first passage of gas was slightly earlier in group B, but did not show a significant difference. However, the length of hospital stay was 6.7 days in group B, which was significantly shorter than that in group $A, 7.3$ days $(P=0.018)$ (Table 2).

Table 1. Demographics of patients

\begin{tabular}{lccc}
\hline Variable & Group A $(n=67)$ & Group B $(\mathrm{n}=65)$ & P-value \\
\hline Age $(\mathrm{yr})$ & $62.6 \pm 11.1$ & $63.3 \pm 11.5$ & 0.730 \\
Sex (male:female) & $36: 31$ & $29: 36$ & 0.295 \\
Body mass index $\left(\mathrm{kg} / \mathrm{m}^{2}\right)$ & $23.5 \pm 4.3$ & $24.3 \pm 3.7$ & 0.238 \\
ASA physical status & & & 0.483 \\
$1-2$ & $64(95.5)$ & $61(93.8)$ & \\
3 & $3(4.5)$ & $4(6.2)$ & \\
History of previous operation & $11(16.4)$ & $14(21.5)$ & 0.284 \\
\hline
\end{tabular}

Values are presented as mean \pm standard deviation or number (\%). ASA, American Society of Anesthesiologists.

\section{DISCUSSION}

Normal gastrointestinal motility is maintained through complex mechanisms involving the central nervous system, gastrointestinal system, hormones, neurotransmitters and inflammatory reactions [8]. Postoperative gastrointestinal motility is normally recovered within several hours for the small intestine, 24-48 hours for the stomach, and 3-5 days for the large intestine [9]. Postoperative ileus is one of the most common complications after abdominal surgery [2]. Although its pathogenic mechanism is still controversial, factors such as overstimulation of the sympathetic nervous system, increased blood catecholamine levels, electrolyte imbalance, irritation of the peritoneum or retroperitoneum, use of narcotic analgesics and type of surgical procedure are thought to contribute to postoperative ileus $[10,11]$.

Recently, the importance of neurohormonal factors has been highlighted, and the splanchnic nerve is known to play an important role in the pathogenic mechanism of ileus [12]. After surgery, vasoactive intestinal polypeptide levels increase, which directly suppresses the contraction of the smooth muscles of the small intestine [13], but the secretion of hormones that promote intestinal motility, such as gastrin, neurotensin and pancreatic polypeptide, decreases [11]. Pain increases the secretion of substance P, which suppresses intestinal motility $[14,15]$, and the use of analgesics affect the enteric nervous system, increasing enteric input and decreasing gastrointestinal motility and peristalsis.

In addition a large surgical site has been shown to lead to severe tissue damage, which, in turn, activates local inflammatory factors, thereby causing ileus [16]. For this reason, the incidence of postoperative ileus in laparoscopic surgery is lower than that in conventional open surgery [3]. Rapid recovery of intestinal motility after abdominal surgery could decrease the length of postoperative hospital stay and the medical costs.

Table 2. Short-term clinical outcomes between the two groups

\begin{tabular}{lccc}
\hline Variable & Group A ( $\mathrm{n}=67)$ & Group B ( $\mathrm{n}=65)$ & P-value \\
\hline Operation time (min), mean \pm SD & $126.9 \pm 34.1$ & $119.41 \pm 35.7$ & 0.218 \\
Blood loss (mL), mean \pm SD & $42.2 \pm 54.2$ & $47.7 \pm 74.2$ & 0.630 \\
Operation title & & & 0.572 \\
Rt or extended Rt colectomy & 23 & 13 & \\
Transverse colectomy & 1 & 1 & \\
Lt or extended Lt colectomy & 3 & 3 & \\
Anterior resection & 25 & 30 & \\
Low anterior resection & 15 & 17 & \\
Total colectomy & 0 & 1 & \\
Diet start (postoperative day) & 2.34 & 2.32 & 0.861 \\
Flatus passing (postoperative day) & 2.47 & 2.29 & 0.266 \\
Hospital stay (postoperative day) & 7.34 & 6.72 & 0.018 \\
\hline
\end{tabular}

Rt, right; Lt, left. 
Gum chewing is a type of "sham feeding" that activates the cephalic-vagal reflex in a manner similar to that when eating food and stimulates the motility of the duodenum, stomach and rectum. Gum chewing increases the serum concentrations of gastrin, neurotensin and pancreatic polypeptide, stimulates the motility of the duodenum, stomach and rectum, and promotes intestinal motility [17-19]. Several studies reported that the first passage of gas was faster and the length of postoperative hospital stay were significantly less in the gum-chewing group; also, rapid recovery of intestinal motility was shown in the gum-chewing group $[6,20]$.

Although we used feeding protocols that commenced with sips of water on the second postoperative day, with the diet being escalated only after passage of flatus, there has been a move toward early postoperative feeding regimes as part of enhanced recovery after surgery (ERAS) program. The ERAS program features a push toward earlier gastrointestinal recovery and discharge, and some patients feel discomfort with ERAS feeding protocol. In this case, we think that gum chewing would lead to improved gut and functional recovery and make patients feel more comfortable.

This study had some limitations. First, although the data were collected prospectively, this study was a retrospective study. Second, the study volume was too small to evaluate the exact effect of sham feeding. A larger scale prospective randomized study is needed to evaluate the exact effect of sham feeding on the recovery from laparoscopic colorectal cancer surgery. Third, the use of analgesics could affect the recovery of intestinal motility, so the effect of analgesics must be taken into consideration [21, 22]. However, all patients in this study used patient-controlled analgesia; unfortunately we were not able to determine the correlation between the use of analgesics and recovery of intestinal motility. Fourth, we recommend patients for discharge according to the criteria discussed above, but some refused to be discharged from the hospital for the reasons other than medical reasons; thus, the length of postoperative hospital stay might have been influenced by that decision.

Gum chewing is extremely easy and very cost-effective. We also think that gum chewing can help prevent dehydration of the mouth. In addition, the process of chewing, the sweet taste, and the smell of gum satisfied the patient's appetite, leading to increased patient comfort.

In conclusion, this study showed that the length of the postoperative hospital stay was shorter in the gum-chewing group, and we think that gum chewing is an easy and cost-effective method to reduce the length of the postoperative hospital stay for laparoscopic colorectal cancer surgery. In future studies, we expect to elucidate the effect of gum chewing on the postoperative recovery more clearly.

\section{CONFLICT OF INTEREST}

No potential conflict of interest relevant to this article was reported.

\section{REFERENCES}

1. Korean Surgical Society. Textbook of surgery. Seoul: Koonja Publisher; 2011.

2. Kehlet H, Holte K. Review of postoperative ileus. Am J Surg 2001; 182(5A Suppl):3S-10S.

3. Milsom JW, Bohm B, Hammerhofer KA, Fazio V, Steiger E, Elson P. A prospective, randomized trial comparing laparoscopic versus conventional techniques in colorectal cancer surgery: a preliminary report. J Am Coll Surg 1998;187:46-54.

4. Choi J, O'Connell TX. Safe and effective early postoperative feeding and hospital discharge after open colon resection. Am Surg 1996;62:853-6.

5. Stewart BT, Woods RJ, Collopy BT, Fink RJ, Mackay JR, Keck JO. Early feeding after elective open colorectal resections: a prospective randomized trial. Aust N Z J Surg 1998;68:125-8.

6. Asao T, Kuwano H, Nakamura J, Morinaga N, Hirayama I, Ide M. Gum chewing enhances early recovery from postoperative ileus after laparoscopic colectomy. J Am Coll Surg 2002;195:30-2.

7. Stern RM, Crawford HE, Stewart WR, Vasey MW, Koch KL. Sham feeding. Cephalic-vagal influences on gastric myoelectric activity. Dig Dis Sci 1989;34:521-7.

8. Luckey A, Livingston E, Tache Y. Mechanisms and treatment of postoperative ileus. Arch Surg 2003;138:206-14.

9. Livingston EH, Passaro EP Jr. Postoperative ileus. Dig Dis Sci 1990;35:121-32.

10. Smiddy FG. The medical management of the surgical patient. Chicago: Year-Book Medical Publishers; 1976.

11. Resnick J, Greenwald DA, Brandt LJ. Delayed gastric emptying and postoperative ileus after nongastric abdominal surgery: part I. Am J Gastroenterol 1997;92:751-62.

12. Holte K, Kehlet H. Postoperative ileus: a preventable event. Br J Surg 2000;87:1480-93.

13. Deloof S, Croix D, Tramu G. The role of vasoactive intestinal polypeptide in the inhibition of antral and pyloric electrical activity in rabbits. J Auton Nerv Syst 1988;22:167-73.

14. Hasler WL. Pharmacotherapy for intestinal motor and sensory disorders. Gastroenterol Clin North Am 2003;32:707-32.

15. Green DW, Gomez G, Greeley GH Jr. Gastrointestinal peptides. Gastroenterol Clin North Am 1989;18:695-733.

16. Kalff JC, Schraut WH, Simmons RL, Bauer AJ. Surgical manipulation of the gut elicits an intestinal muscularis inflammatory response resulting in postsurgical ileus. Ann Surg 1998;228:652-63.

17. Soffer EE, Adrian TE. Effect of meal composition and sham feeding on duodenojejunal motility in humans. Dig Dis Sci 1992;37: 1009-14.

18. Jepsen JM, Skoubo-Kristensen E, Elsborg L. Rectosigmoid motility response to sham feeding in irritable bowel syndrome: evidence of a cephalic phase. Scand J Gastroenterol 1989;24:53-6.

19. Stern RM, Crawford HE, Stewart WR, Vasey MW, Koch KL. Sham feeding. Cephalic-vagal influences on gastric myoelectric activity. Dig Dis Sci 1989;34:521-7. 
20. Schuster R, Grewal N, Greaney GC, Waxman K. Gum chewing reduces ileus after elective open sigmoid colectomy. Arch Surg 2006;141:174-6.

21. Bardon T, Ruckebusch Y. Comparative effects of opiate agonists on proximal and distal colonic motility in dogs. Eur J Pharmacol
1985;110:329-34.

22. Yukioka H, Bogod DG, Rosen M. Recovery of bowel motility after surgery: detection of time of first flatus from carbon dioxide concentration and patient estimate after nalbuphine and placebo. Br J Anaesth 1987;59:581-4. 